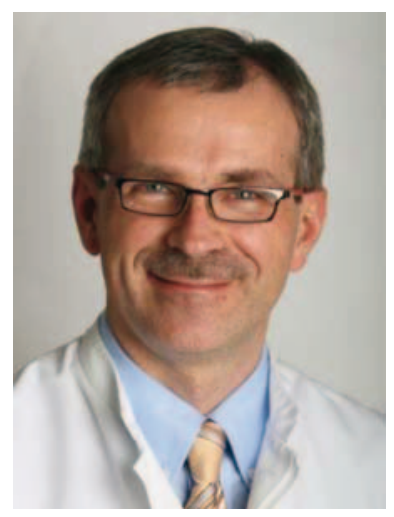

Dr. med. Cesar Winnicki, Brunnen

\section{Der wichtige Schritt in der Onkologie}

Das renommierte Journal of Clinical Oncology begann im Januar 1998 sein Editorial mit einem provokativen Titel: «Alternative Medizin - ein attraktiver Unsinn». Im darauf folgenden Text hat die Redaktion ihre ablehnende Haltung gegenüber der Komplementärmedizin ausführlich dargestellt und begründet. Wie überrascht mussten die Leser dieser Fachzeitschrift sein, als sich bereits nach zwei Jahren das Blatt wendete und der komplementäre Ansatz seriös präsentiert wurde. «Komplementäre Therapieverfahren mit Krebspatienten diskutieren: Worüber sollen wir sprechen?» - mit diesem Titel lancierte die Redaktion der Zeitschrift eine konstruktive Diskussion über komplementäre Verfahren in der Onkologie. "Der attraktive Unsinn» hat Onkologen in den USA so beschäftigt und fasziniert, dass es im Jahr 2003 zur Gründung einer Gesellschaft für Integrative Onkologie kam. Im November dieses Jahres hat bereits zum siebten Mal ein internationaler Kongress für Integrative Onkologie in New York stattgefunden.

\section{Worüber sollen wir sprechen?}

Das Jahr 2002 sei das Wendejahr der Onkologie. In den USA sind die Krebsraten zum ersten Mal seit Jahrzehnten gesunken und gehen um durchschnittlich $2 \%$ pro Jahr zurück. Generell werden alle Tumorerkrankungen früher diagnostiziert und gezielter behandelt. Die moderne Onko- logie hat in den letzten Jahren einen immensen Fortschritt im Verständnis der molekularen Mechanismen der Karzinogenese erzielt. Diese Erkenntnisse führen wiederum zu innovativen Therapieansätzen. Die individuelle onkologische Betreuung wird die Schicksale der Betroffenen künftig wesentlich verändern.

\section{Kehrseite der Medaille}

Wenn man die aktuelle onkologische Statistik in etwas breiterem Kontext betrachtet, ergibt sich ein weniger optimistisches Bild. Vor 30 Jahren betrug die 5-Jahres-Überlebensrate aller onkologischen Patienten in Deutschland $42 \%$, im 2003 stieg sie auf 55\% an [1]. Bei fortgeschrittenen Tumorstadien hat sich die Lage nur unwesentlich verändert - z.B. bei metastasiertem Brustkrebs wurde seit 20 Jahren keine Lebensverlängerung erreicht [2]. Dieser eher bescheidene Fortschritt muss unbedingt in das richtige Verhältnis zu den Kosten der onkologischen Betreuung gebracht werden. Neulich hat sich die Zeitschrift der amerikanischen Ärztegesellschaft mit diesem Thema auseinandergesetzt [3]. 1990 hat die gesamte onkologische Betreuung die Amerikaner 27 Milliarden Dollar gekostet; 2008 waren es bereits 90 Milliarden. Nun scheint es, dass sogar das amerikanische Gesundheitssystem in der Finanzierung der Onkologie an seine Grenzen stösst. Bei dieser vielleicht etwas polemischen Betrachtung dürfen wir nicht vergessen, dass wir uns auf einige wenige wohlhabende Industrienationen beschränken. Weltweit ist die Krebsepidemiologie nicht anders als dramatisch zu bezeichnen. Die Weltgesundheitsorganisation WHO schätzt aktuell weltweit die Anzahl der onkologischen Neuerkrankungen auf 10 Millionen pro Jahr und rechnet mit einer Zunahme auf 15 Millionen im Jahr 2020. Für diese massive Zunahme seien zwei Faktoren verantwortlich. Einerseits die höhere Lebenserwartung der Bevölkerung, andererseits die exogenen und somit vermeidbaren Faktoren. Laut WHO wird die Entstehung von Krebserkrankungen zu 60\% durch die Umweltfaktoren und einen falschen Lebensstil beeinflusst. Diese beunruhigende Feststellung muss künftig in der Gesundheitspolitik unbedingt berücksichtigt werden. Mit anderen Worten: Ein Grossteil der Krebserkrankungen kann durch präventive Massnahmen vermieden werden. Komplementärmedizin hat diesen Ansatz bereits vor Jahren entdeckt und setzt ihn praktisch um.

\section{KARGER}

Fax +497614520714 Information@Karger.de www.karger.com
(๑) 2010 S. Karger GmbH, Freiburg

Accessible online at: www.karger.com/szg
Dr. med. Cesar Winnicki

Chefarzt Aeskulap-Klinik

Gersauerstrasse 8, 6440 Brunnen, Schweiz

Tel. +41 41 825-4747, Fax -4965

Cesar.Winnicki@aeskulap.com 


\section{Der mündige Patient}

Der immense Fortschritt und Wandel der Medizin in den letzten Jahrzehnten ging an unseren Patienten nicht spurlos vorbei. «Der bevormundete Patient im ärztlichen $\mathrm{Pa}$ triarchat» (G. Nagel) wurde allmählich zu einem mündigen, aufgeklärten Partner des Arztes. Auf der einen Seite wissen unsere Patienten immer mehr über ihre Krankheit und deren Therapie und haben dadurch oft überhöhte Erwartungen gegenüber der Heiltechnik. Andererseits wollen immer mehr Patienten die Verantwortung für ihre Gesundheit übernehmen sowie bewusst und aufgeklärt mit den vorgeschlagenen Massnahmen umgehen. Gerade in der komplexen onkologischen Behandlung ist der Wunsch nach einem individuellen, partnerschaftlichen Vorgehen sehr aktuell. Wie das Beispiel des Prostatakarzinoms exemplarisch zeigt, ist die kontrollierte Beobachtung und Zurückhaltung gegenüber den invasiven Massnahmen immer häufiger die beste Option. Diese «Watchful Waiting»-Strategie setzt ein hohes Mass an gegenseitigem Vertrauen und reibungsloser Kommunikation zwischen Arzt und Patient voraus und trägt gleichzeitig den individuellen Gegebenheiten und Bedürfnissen unserer Patienten Rechnung. Der heutige Krebspatient sucht nicht nur ein optimales Therapiekonzept oder eine möglichst hochqualitative «Reparatur», sondern fragt nach dem Sinn der Erkrankung, geht alternative Wege, möchte einen persönlichen Beitrag zur Erhaltung der Gesundheit leisten. Der traditionelle Fokus der Mediziner auf die Organerkrankung (Pathogenese) wird durch ganzheitliche Aspekte ergänzt, in deren Mittelpunkt Gesundheitsförderung und eigene Ressourcen stehen (Salutogenese). Komplementärmedizin bietet diesen autonomen Patienten Verständnis für ihre Auffassung und unterstützt sie bei der Umsetzung nötiger Massnahmen. Das macht die Komplementärmedizin so populär und gefragt.

\section{Komplementäre Onkologie - mehr als nur Methoden}

Komplementieren heisst aus dem Lateinischen «ergänzen/ vervollständigen». In diesem Sinne wird zunehmend die Rolle biologischer Methoden in der Onkologie verstanden. Es geht um eine harmonische, individuelle Ergänzung der standardmässigen Therapien der Schulmedizin durch bewährte Massnahmen der Komplementärmedizin. Dieser duale, integrative Ansatz ist für beide Exponenten des Heilungsprozesses, d.h. sowohl Arzt als auch Patient, optimal. Die Methoden der Komplementärmedizin wer- den nicht anstelle von Chemo- oder Strahlentherapie eingesetzt, sondern haben einen supportiven, ergänzenden Charakter. Durch die Integration beider Systeme kann das Maximum für die körperliche wie auch psychisch-geistige Verfassung des Patienten erreicht werden. Aus der bisherigen Erfahrung der Integrativen Onkologie können folgende Schwerpunkte für diesen Ansatz herauskristallisiert werden:

- Prävention der Tumorerkrankungen vor allem durch Unterstützung der Lebensstilveränderungen und Vorsorgeuntersuchungen.

- Komplementärmedizinische Begleittherapie bei Standardmassnahmen der Onkologie mit dem Ziel, die Nebenwirkungen dieser Therapien zu reduzieren und einen synergistischen Effekt in Bezug auf die Wirksamkeit $\mathrm{zu}$ erreichen.

- Unterstützung der Rekonvaleszenz und der Sekundärprophylaxe nach erfolgten onkologischen Behandlungen mit dem Ziel, die Rezidivquote zu reduzieren.

- Begleitung und Unterstützung der Patienten in terminalen Phasen der Erkrankung (Palliative Care).

Auf diesen Gebieten sollte die Schulmedizin mit der Komplementärmedizin Hand in Hand zusammenarbeiten, um den wachsenden Herausforderungen des Gesundheitswesens besser gerecht $\mathrm{zu}$ werden. Es freut, festzustellen, dass der integrative Ansatz in der Schweiz bereits praktisch umgesetzt wird. Vor einem Jahr wurde das Thema Integrative Onkologie anlässlich eines Symposiums im Kantonsspital St. Gallen zum ersten Mal präsentiert und intensiv diskutiert. Vor kurzem folgte eine Tagung in der Aeskulap-Klinik in Brunnen, in der neben den theoretischen Aspekten die praktische Umsetzung des integrativen Konzepts dargestellt wurde. Bei diesen Anlässen wurde die Komplementärmedizin als wertvoller Teil moderner ganzheitlicher Krebsbehandlung betrachtet. Es scheint, dass damit ein wichtiger Schritt in die Neuorientierung der patientenzentrierten onkologischen Betreuung gesetzt wurde.

Dr. med. Cesar Winnicki, Brunnen

\section{Literatur}

1 Brenner H, et al.: Verbesserte Langzeitüberlebensraten von Krebspatienten Die unterschätzten Fortschritte der Onkologie. Dtsch Arztebl 2005;102:A 2628-2633.

2 Schlesinger-Raab A, et al.: Metastasiertes Mammakarzinom: Keine Lebensverlängerung seit 20 Jahren. Dtsch Arztebl 2005;102:A 2706-2714.

3 Elkin EB, Bach PB: Cancer's next frontier: addressing high and increasing costs. JAMA 2010;303:1086-1087. 